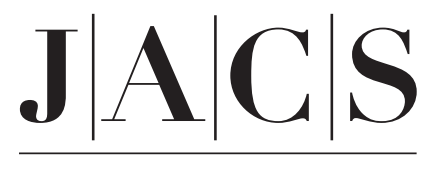

A R T I C L E S

Published on Web 11/15/2002

\title{
The Role of Surface Free Energy on the Formation of Hybrid Bilayer Membranes
}

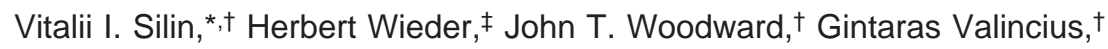 \\ A. Offenhausser, $\$$ and Anne L. Plant ${ }^{\dagger}$ \\ Contribution from the National Institute of Standards and Technology, Biotechnology Division, \\ Gaithersburg, Maryland 20899, FRIZ Biochem GmbH, 82152 Martinsried, Germany, and \\ Institute for Thin Films and Interfaces, Research Center Juelich D-52425 Juelich, Germany
}

Received April 18, 2002

\begin{abstract}
The interaction of small phospholipid vesicles with well-characterized surfaces has been studied to assess the effect of the surface free energy of the underlying monolayer on the formation of phospholipid/ alkanethiol hybrid bilayer membranes (HBMs). The surface free energy was changed in a systematic manner using single-component alkanethiol monolayers and monolayers of binary mixtures of thiols. The binary surfaces were prepared on gold by self-assembly from binary solutions of the thiols $\mathrm{HS}-\left(\mathrm{CH}_{2}\right)_{n}-\mathrm{X}(n=$ $11, \mathrm{X}=\mathrm{CH}_{3}$ or $\mathrm{OH}$ ) in THF. Surface plasmon resonance (SPR), electrical capacitance, and atomic force microscopy (AFM) measurements were used to characterize the interaction of palmitoyl,oleoyl-phosphatidylcholine (POPC) vesicles with the surfaces. For all surfaces examined, it appears that the polar part of surface energy influences the nature of the POPC assembly that associates with the surface. Comparison of optical, capacitance, and AFM data suggests that vesicles can remain intact or partially intact even at surfaces with a contact angle with water of close to $100^{\circ}$. In addition, comparison of the alkanethiols of different chain lengths and the fluorinated compound $\mathrm{HS}-\left(\mathrm{CH}_{2}\right)_{2}-\left(\mathrm{CF}_{2}\right)_{8}-\mathrm{CF}_{3}$ that characterize with a low value of the polar part of the surface energy suggests that the quality of the underlying monolayer in terms of number of defects has a significant influence on the packing density of the resulting HBM layer.
\end{abstract}

\section{Introduction}

Supported lipid membranes enable biofunctionalization of inorganic, polymer, and conducting surfaces and provide a natural environment for protein immobilization under nondenaturing conditions and in a well-defined orientation. ${ }^{1}$ Understanding the forces that direct the self-assembly of phospholipid molecules in predictable arrangements will aid a development of applications based on phospholipid molecules self-assembly in sensors, diagnostics, biomimetic materials, and biological arrays where they may incorporate features such as molecular recognition and enzymatic activity, will help for cell membrane applications in the development of the biological assays for transmembrane proteins, ${ }^{2}$ and applications in the emerging field of proteomics.

Supported lipid bilayers ${ }^{1}$ can be formed from vesicles interacting with glass, silica, or mica $^{3-7}$ or by the interaction

* To whom correspondence should be addressed. Tel.: (301) 975-4509. Fax: (301) 975-8246. E-mail: vitalii.silin@ nist.gov.

$\dagger$ NIST.

FRIZ Biochem GmbH.

$\$$ Institute for Thin Films and Interfaces.

(1) Sackmann, E. Science 1996, 271, 43-48.

(2) Rao, N. M.; Silin, V. I.; Ridge, K. D.; Woodward, J. T.; A. L., P. Anal. Biochem. 2002, 307, 117-130.

(3) Brian, A. A.; McConnell, H. M. Proc. Natl. Acad. Sci. U.S.A. 1984, 81, 6159-6163.

(4) Cremer, P. S.; Boxer, S. G. J. Phys. Chem. B 1999, 103, 2554-2559.

(5) Nollert, P.; Kiefer, H.; Jahnig, F. Biophys. J. 1995, 69, 1447-1455.

(6) Radler, J.; Strey, H.; Sackmann, E. Langmuir 1995, 11, 4539-4548.

(7) Tamm, L. K.; McConnell, H. M. Biophys. J. 1985, 47, 105-113.

14676 a J. AM. CHEM. SOC. 2002, 124, 14676-14683 of vesicles with a hydrophobic film formed on glass using silanes ${ }^{8-10}$ or on metal using alkanethiols ${ }^{11-14}$ or thiolipid..$^{15-17}$ In general, the curvature, the osmotic pressure, and the lateral tension of the phospholipid vesicles, the concentration of vesicles, and the adhesion free energy of the surface ${ }^{18,19}$ influence the formation of the phospholipid bilayer on a hydrophilic surface. Lipid vesicles will also interact with hydrophobic surfaces and add a layer of phospholipid to form a bilayer, but the details of this process are poorly understood. Kalb et al. ${ }^{20}$ proposed a mechanism, which suggested how a stable vesicle might delaminate on a hydrophobic surface. Hubbard et al. ${ }^{21}$ demonstrated the influence of DMPC vesicle

(8) Miller, C.; Cuendet, P.; Gratzel, M. J. Electroanal. Chem. 1990, 278, 175192.

(9) Terrettaz, S.; Vogel, H.; Gratzel, M. J. Electroanal. Chem. 1992, 326, 161176.

(10) Merkel, R.; Sackman, E.; Evans, E. J. Phys. (Paris) 1989, 50, 1535-1555.

(11) Plant, A. L. Langmuir 1993, 9, 4.

(12) Plant, A. L.; Gueguechkeri, M.; Yap, W. Biophys. J. 1994, 67, 11261133 .

(13) Meuse, C. W.; Krueger, S.; Majkrzak, C. F.; Dura, J. A.; Fu, J.; Conner, J. T.; Plant, A. L. Biophys. J. 1998, 74, 1388.

(14) Meuse, C. W.; Niaura, G.; Lewis, M. L.; Plant, A. L. Langmuir 1998, 14 1604.

(15) Terrettaz, S.; Stora, T.; Duschl, C.; Vogel, H. Langmuir 1993, 9, 13611369.

(16) Lang, H.; Duschl, C.; Gratzel, M.; Vogel, H. Thin Solid Films 1992, 210 $818-821$

(17) Lingler, S.; Rubinstein, I.; Knoll, W.; Offenhausser, A. Langmuir 1997, 13, 7085-7091.

(18) Lipowsky, R. Nature 1991, 349, 475-481.

(19) Lipowsky, R.; Seifert, U. Mol. Cryst. Liq. Cryst. 1991, 202, 17-25.

(20) Kalb, E.; Frey, S.; Tamm, L. K. Biophys. Biochim. Acta 1992, 1103, $307-$ 316. 
concentration on the kinetics of vesicle reorganization at hydrophobic, hexadecanethiol monolayer surfaces. This study found that the kinetics of bilayer formation strongly depended on the concentration of lipid vesicles, and it presented a model that explained the kinetics as either a diffusion controlled or a kinetically controlled process depending on the vesicle concentration. Keller and Kasemo ${ }^{22}$ studied vesicle adsorption using the quartz crystal microbalance technique on different surfaces, and they found that monolayers of lipid add to a hydrophobic surface, bilayers of lipid add to a hydrophilic glass surface, and intact vesicles are retained on an oxidized gold surface. Furthermore, the technique provided evidence that the addition of lipid bilayers to hydrophilic surfaces was preceded by vesicle adsorption to the surface, but that vesicle adsorption was not observed at hydrophobic surfaces. Evans et al. ${ }^{23}$ examined monolayers of oligoethyleneglycol-supported thio-cholesterol and the appropriate thio-oligoethyleneglycol. They studied the interaction of eggPC vesicles with the surface, and the addition of a phospholipid layer, as a function of the molar fraction of cholesterol at the surface. They found that a single layer of PC added to the hydrophobic cholesterol film and that a bilayer of lipid added to the mixed thiols surfaces.

The focus of this work is to systematically study the influence of the surface free energy, composition, and structure of the underlaying monolayer on vesicle adsorption and rupture at a surface, while keeping constant the size, composition, osmotic pressure, and concentration of the vesicles, and keeping constant the roughness of the surfaces. We used two systems for our experiments: a binary mixture of polar plus nonpolar alkylthiols, which allowed us to tune surface free energy in a systematic manner, and hydrophobic surfaces of alkanethiols of different chain lengths and composition to cover the widest possible range of contact angles. We chose a binary system of a hydrophilic and a hydrophobic alkanethiol of similar chain length, dodecanethiol (DDT) and hydroxyundecanethiol (HUT), to avoid the effects of molecular roughness and phase separation. In addition, self-assembled monolayers of this mixture have been well characterized by others, and methods for preventing phase separation of the components have been determined. ${ }^{23-26}$ The interaction of lipid vesicles with these surfaces was characterized by the combined use of the surface plasmon resonance technique for measuring the optical thickness of the assembly, impedance spectroscopy (IS) for calculating the electrical capacitance of the surface layers, and atomic force microscopy (AFM) to confirm the structure of the POPC layers. Contact angle measurements were used to calculate the surface free energy of the samples. We found that even at surfaces with large contact angles with water $\left(>90^{\circ}\right)$, vesicle fusion with the surface can be arrested.

\section{Experimental Section}

Certain commercial materials, instruments, and equipment are identified in this manuscript to specify the experimental procedure as completely as possible. In no case does such identification imply a

(21) Hubbard, J. B.; Silin, V.; Plant, A. L. Biophys. Chem. 1997, 75, 163-176.

(22) Keller, C. A.; Kasemo, B. Biophys. J. 1998, 75, 1397-1402.

(23) Evans, S. D.; Sharma, R.; Ulman, A. Langmuir 1991, 7, 146-161

(24) Ulman, A.; Evans, S. D.; Shnidman, Y.; Sharma, R.; Eilers, J. E.; Chang, J. C. J. Am. Chem. Soc. 1991, 113, 1499-1506.

(25) Ulman, A.; Evans, S. D.; Shnidman, Y.; Sharma, R.; Eilers, J. E. Adv Colloid Interface Sci. 1992, 39, 175-224.

(26) Ulman, A. Thin Solid Films 1996, 273, 48-53. recommendation or endorsement by the National Institute of Standards and Technology, nor does it imply that the materials, instruments, or equipment identified are necessarily the best available for the purpose.

Preparation of Gold Films and Alkanethiol Monolayers. Highquality glass substrates (size $14 \times 25 \times 1.5 \mathrm{~mm}$ ) were coated with a thin layer of chromium and then with gold for SPR and capacitance measurements. Before metal coating, samples were washed in detergent solution, rinsed in water, and incubated in Nochromix $(125 \mathrm{~g} / \mathrm{L}$ of potassium persulfate in concentrated sulfuric acid) solution for 30-45 min. After being extensively rinsed in high purity water (resistivity 18.2 $\mathrm{M} \Omega$ ), samples were washed with high purity acetone and dried under a stream of nitrogen gas. A high vacuum system (Edwards 306, West Sussex, U.K.) equipped with two magnetron sputtering sources (one for chromium, another one for the gold) and a dc power source (MDX-1K, Advanced Energy Industries, Fort Collins, CO) was used for metal film deposition on the clean glass substrates. The power for gold deposition was $750 \mathrm{~W}$ and for chromium $440 \mathrm{~W}$. The basic vacuum level was at least $1 \times 10^{-5} \mathrm{~Pa}\left(1 \times 10^{-7} \mathrm{mbr}\right)$. The argon pressure $\left(0.6 \mathrm{~Pa}\left(6 \times 10^{-3} \mathrm{mbr}\right)\right)$ during the sputtering process was kept constant using a "butterfly" valve (MKS Type 250, MKS Instruments, Andover, MA). During the sputtering process, samples were rotating over the gold/chromium targets (Superior Electric SLO-SXN stepper motor, Warner Electronic, Bristol, CT). A specially shaped mask was placed between the target and the sample holder to ensure homogeneity of film thickness. A time per one rotation of the samples over gold/chromium targets was calibrated versus metal film thickness using X-ray reflectivity and neutron scattering. The thickness of the chromium sublayer was $2 \mathrm{~nm} \pm 2 \%$, and the gold film was $49 \mathrm{~nm} \pm$ $1 \mathrm{~nm}$ thick. AFM indicated that gold films prepared in this way on freshly cleaned samples had a surface roughness of less than $3 \AA$ rms. The homogeneity of the film thickness as measured using light transmission was better than $3 \%$ on $100 \mathrm{~mm}$ (4 in) samples.

For electrochemical measurements, gold with the same thickness was deposited onto silicon wafers $(\langle 100\rangle$, N-Type; Virginia Semiconductor, Fredericksburg, VA) using the same protocol as for the glass substrates. The thickness of the chromium sublayer was $2 \mathrm{~nm} \pm 2 \%$, and the gold film was $49 \mathrm{~nm} \pm 1 \mathrm{~nm}$ thick. Immediately after metal deposition, the gold-coated samples were immersed into binary solutions of alkylthiols (final concentration of $2 \mathrm{mM}$ total thiol in freshly distilled THF) for $24 \mathrm{~h}$, after which they were rinsed with THF and stored in THF. Because previously published data ${ }^{24-26}$ indicate that the HUT/ DDT surface is not completely stable and changes slowly with the time in the ambient laboratory environment, we used our surfaces immediately after preparation. For single-component alkanethiol monolayers, $1 \mathrm{mM}$ solutions of octadecanethiol (C8), dodecanethiol (C12), hexadecanethiol (C16), octadecanethiol (C18), and the fluorinated compound, HS $-(\mathrm{CH} 2) 2-(\mathrm{CF} 2) 8-\mathrm{CF} 3(\mathrm{CF})$ were prepared in THF. Freshly prepared gold-coated silicon samples were placed in solutions for 24 $\mathrm{h}$, followed by extensive rinsing with THF, and were used immediately.

Vesicle Preparation. Small unilamellar vesicles were prepared by the 2-propanol injection method. ${ }^{27}$ First $2 \mu \mathrm{mol}$ of the lipid, palmitoyloleoyl-phosphatidylcholine (POPC, Avanti Polar Lipids, Alabaster, AL), was dissolved in $50 \mu \mathrm{L}$ of 2-propanol and injected with a syringe into $1 \mathrm{~mL}$ of phosphate buffer $(50 \mathrm{mM}, \mathrm{pH} 7.4)$ while vortexing. The resulting dispersion was diluted by a factor of 10 with buffer solution and degassed under vacuum. This procedure resulted in a dispersion of unilamellar vesicles with a diameter of $58 \pm 12 \mathrm{~nm}(95 \%$ of the population in the range of $56-60 \mathrm{~nm}$ ), as determined by quasi-elastic light scattering (Coulter Submicron Particle Analyzer Model N4MD, Hialeah, FL), and a final concentration of $0.2 \mathrm{mM}$ in lipid.

Surface Plasmon Resonance. SPR measurements were carried out on a device described previously. ${ }^{28}$ Association of POPC with the alkylthiol monolayers was monitored under nonflow conditions.

(27) Batzri, S.; Korn, E. D. Biochim. Biophys. Acta 1973, 901, 157.

(28) Silin, V.; Weetall, H.; Vanderah, D. J. J. Colloid Interface Sci. 1997, 185, 94-103. 
Vesicles were added to the cell with an automatic pipet, by rapidly (1-2 s) replacing all buffer solution in the cell with buffer solution containing vesicles. At the end of the incubation period, buffer only was allowed to flow into the cell to remove the vesicle solution, keeping the surface under the buffer during the wash period. The SPR cell volume was $0.9 \mathrm{~mL}$, the experiments were performed at a temperature of $22.4^{\circ} \mathrm{C}$, and the sampling rate was $2 \mathrm{~s}$ with a $0.02 \mathrm{~s}$ CCD exposure time.

Impedance Spectroscopy. Impedance spectra were collected from $50 \mathrm{kHz}-100 \mathrm{mHz}$ with a frequency response analyzer (Solartron 1250, EG\&G, Princeton, NJ) combined with a potentiostat (Solartron 1286). Spectra were taken before vesicle addition to the cell, and during and after lipid association with the surface by superimposing an alternating voltage amplitude of $10 \mathrm{mV}$ (rms) on the open circuit potential.

The capacitance of the samples was measured in two dc polarization modes: (i) open circuit potential (OCP) and (ii) $E=0.0 \mathrm{~V}$ vs $\mathrm{Ag} /$ $\mathrm{AgCl}, \mathrm{KCl}$ (sat). In both cases, the IS spectra were the same within measurement uncertainties.

The model (equivalent circuit) that was used to estimate the capacitance of both the monolayers and the adsorbed lipid was a simple series $R C$ circuit, in which $R$ was the solution resistance, and $C$ was the capacitance of the sample. We used this model because in the Nyquist complex capacitance plot (Im $Y / \omega$ vs Re $Y / \omega$, where $Y$ is the admittance, and $\omega$ is the cyclic frequency), we observed an almost perfect semicircular shape of the EIS spectrum, which is characteristic of ideal $R C$ behavior. To assess the extent to which the samples behaved as ideal capacitors, we added a constant phase element to the capacitance in the model. Fitting of the experimental spectra to the model yielded the constant phase exponent value within the interval from 0.98 to 1.00 for all samples. As a value of 1 is indicative of ideal capacitive behavior, this allowed us to assume an approximate equality $C=$ constant phase element coefficient. The fitting procedure yielded $\chi$-squared function values from approximately $0.8 \mathrm{e}^{-4}$ to $3 \mathrm{e}^{-4}$, indicating a good fit quality.

Reductive Desorption Experiment. The sample was placed into an electrochemical cell, then covered with $0.5 \mathrm{M} \mathrm{NaOH}$ and bubbled with argon for at least $15 \mathrm{~min}$. The reductive desorption was carried out using a potentiostat (Solartron 1286) by running a cyclic voltammogram from 0 to $-1.25 \mathrm{~V}$ ( $\mathrm{vs} \mathrm{Ag} / \mathrm{AgCl} / 3 \mathrm{M} \mathrm{NaCl}$ ) with a scan speed of $100 \mathrm{mV} / \mathrm{s}$.

Measurement of Surface Free Energy Using Contact Angle Data. The method of Owens and Wendt ${ }^{29}$ was used to determine the polar and dispersive component of the surface free energy by measuring the contact angles formed with two solvents: water and diiodomethane. Contact angles were determined with a goniometer (Rame-Hart model 110-00-115, Mountain Lakes, NJ) at room temperature and ambient $(\sim 30 \%)$ relative humidity using water and diiodomethane as probing liquids. Sessile drop contact angles were measured by lowering an $\sim 2$ $\mu \mathrm{L}$ drop of the liquid onto the surface from a needle tip. The data represent an average of at least five measurements that were taken for each surface.

Atomic Force Microscopy. Samples were imaged using the PicoScan AFM (Molecular Imaging; Phoenix, AZ) in intermittent contact mode using a $7 \mu \mathrm{m}$ scan head. POPC vesicles were added in buffer solution into the fluid cell, and samples were imaged in the fluid cell in the presence of vesicle solution.

\section{Results}

Characterization of the Single Alkanethiol and Binary Alkanethiol/Hydroxythiol Monolayer Surfaces. Surface Free Energy Data. Binary alkylthiol monolayers containing a mixture of hydrophilic $\left(\mathrm{OH}\right.$-terminal) plus hydrophobic $\left(\mathrm{CH}_{3}\right.$-terminal $)$ groups were first engineered and studied in detail by Ulman ${ }^{24-26}$ with the goal of fine-tuning the surface free energy. To be sure

(29) Owens, D. K.; Wendt, R. C. J. Appl. Polym. Sci. 1969, 13, 1741-1747.
Table 1. Advancing Contact Angles for Water $\left(\theta_{\mathrm{W}}\right)$ and lodomethane $\left(\theta_{1}\right)$ and Calculated Dispersive $\left(\gamma_{\mathrm{S}}{ }^{\mathrm{d}}\right)$, Polar $\left(\gamma_{\mathrm{S}}{ }^{\mathrm{p}}\right)$, and Total $\left(\gamma_{s}\right)$ Surface Energies of the Binary Alkyl Thiol Layers versus DDT Concentration $\left(X_{\mathrm{DDT}}\right)$ in the Binary HUT/DDT Layers

\begin{tabular}{lrrrrrrrrrr}
\hline$X_{\text {DDT }}$ & 1 & 0.95 & 0.9 & 0.8 & 0.7 & 0.6 & 0.5 & 0.4 & 0.2 & 0.0 \\
$\theta_{\mathrm{W}}$ & 109.5 & 103.6 & 101.2 & 95.7 & 87.2 & 72.4 & 57.9 & 47.9 & 40.1 & 33.5 \\
$\theta_{\mathrm{I}}$ & 69.7 & 68.3 & 66.4 & 62.5 & 58.8 & 52.2 & 48.7 & 41.9 & 36.5 & 34.1 \\
$\gamma_{\mathrm{s}}{ }^{\mathrm{d}}, \mathrm{mJ} / \mathrm{m}^{2}$ & 23.0 & 22.9 & 23.8 & 25.4 & 26.3 & 28.0 & 27.8 & 30.1 & 31.8 & 32.2 \\
$\gamma_{\mathrm{s}} \mathrm{p}, \mathrm{mJ} / \mathrm{m}^{2}$ & 0.2 & 0.9 & 1.2 & 2.2 & 4.6 & 10.9 & 19.8 & 25.1 & 29.0 & 32.5 \\
$\gamma_{\mathrm{S}}, \mathrm{mJ} / \mathrm{m}^{2}$ & 23.2 & 23.8 & 25.0 & 27.6 & 30.9 & 38.9 & 47.6 & 55.2 & 60.8 & 64.7
\end{tabular}

Table 2. Advancing Contact Angles for Water $\left(\theta_{\mathrm{W}}\right)$ and lodomethane $\left(\theta_{1}\right)$ and Calculated Dispersive $\left(\gamma_{s}{ }^{d}\right)$, Polar $\left(\gamma_{s}^{p}\right)$, and Total $\left(\gamma_{\mathrm{S}}\right)$ Surface Energies of the Pure Hydrophobic Alkyl Thiol Layers: $\mathrm{CF} 8, \mathrm{HS}-\left(\mathrm{CH}_{2}\right)-\left(\mathrm{CF}_{2}\right)_{8}-\mathrm{CF}_{3} ; \mathrm{C} 18, \mathrm{HS}-\left(\mathrm{CH}_{2}\right)_{17}-\mathrm{CH}_{3} ; \mathrm{C} 16$, $\mathrm{HS}-\left(\mathrm{CH}_{2}\right)_{15}-\mathrm{CH}_{3} ; \mathrm{C} 12, \mathrm{HS}-\left(\mathrm{CH}_{2}\right)_{11}-\mathrm{CH}_{3} ; \mathrm{C} 8, \mathrm{HS}-\left(\mathrm{CH}_{2}\right)_{7}-\mathrm{CH}_{3}$

\begin{tabular}{lcrrrr}
\hline \multicolumn{1}{c}{ thiols } & \multicolumn{1}{c}{ CF8 } & \multicolumn{1}{c}{ C18 } & \multicolumn{1}{c}{ C16 } & \multicolumn{1}{c}{ C12 } & \multicolumn{1}{c}{ C8 } \\
\hline$\theta_{\mathrm{W}}$ & 116.4 & 114.8 & 114.3 & 109.5 & 102.3 \\
$\theta_{\mathrm{I}}$ & 75.8 & 76.6 & 79.8 & 69.7 & 66.6 \\
$\gamma_{\mathrm{s}}{ }^{\mathrm{d}}, \mathrm{mJ} / \mathrm{m}^{2}$ & 20.1 & 19.4 & 17.4 & 23.0 & 23.8 \\
$\gamma_{\mathrm{s}}{ }^{\mathrm{p}}, \mathrm{mJ} / \mathrm{m}^{2}$ & 0.01 & 0.1 & 0.2 & 0.2 & 1.0 \\
$\gamma_{\mathrm{S}}, \mathrm{mJ} / \mathrm{m}^{2}$ & 20.1 & 19.4 & 17.6 & 23.2 & 24.8 \\
\hline
\end{tabular}

that our surfaces had the same characteristics as those of Ulman, we measured the surface tension, $\gamma_{\mathrm{L}}$, using the method of Owens and Wendt. ${ }^{29}$ Water and diiodomethane were chosen for contact angle measurements because they differ the most in their $\left(\gamma_{\mathrm{L}} \mathrm{p} /\right.$ $\left.\gamma_{L}^{d}\right)^{1 / 2}$ value, where $\gamma_{L}^{p}$ is the polar part, and $\gamma_{L}{ }^{d}$ is the dispersive part of the surface energy. The surface energy measurement data are shown in Table 1 and are in very good agreement with published data. ${ }^{24-26}$ The polar part of the surface energy changes significantly with the increase of HUT molar fraction in the monolayer, while the dispersive part is almost constant over the whole HUT molar fraction range. At a low concentration of the DDT $\left(X_{\mathrm{DDT}}\right)$, our absolute values differ slightly from those reported, ${ }^{25}$ most likely due to contamination or surface reorganization as has been discussed. ${ }^{26}$ As was shown by Ulman and co-workers ${ }^{24-26}$ for these surfaces: (i) the surface energy can be fine-tuned by changing the relative surface concentration of the DDT and HUT species; (ii) the ratio of the surface concentration of the DDT and HUT corresponds closely to the bulk concentration ratio of these compounds in solvent as long as THF is chosen as the solvent; and (iii) the distribution of the DDT and HUT species on the surface prepared from THF solution is random, meaning that the species are homogeneously mixed and there is no phase separation.

In this study, we also examined single-component surfaces prepared with methyl or fluorocarbon-formulate alkanethiols. The surface energies of the series of single-component alkanethiol monolayers are shown in Table 2. Like the binary surfaces, the variation in the dispersive part of the surface energy as a function of thiol composition is small (less than 15\%). At the same time, the difference in the polar part of the surface energy is relatively large. For example, the ${\gamma_{\mathrm{L}}}^{\mathrm{p}}$ of $\mathrm{C} 12$ is $20 \%$ of the value for $\mathrm{C} 8$, and the value of the fluorinated compound CF is effectively zero. The value $\gamma_{\mathrm{L}}^{\mathrm{p}}$ of C8 is similar to $\gamma_{\mathrm{L}}^{\mathrm{p}}$ of the mixed DDT/HUT surface with $X_{\mathrm{DDT}}=0.9$.

Capacitance Data. The capacitance of the single-component and binary SAM surfaces was determined using impedance spectroscopy. The capacitance data are presented in Figure 1 as a function of the number of carbon atoms in single-component alkanethiol monolayers, and as a function of the relative surface concentration of DDT molecules for binary surfaces. Our 


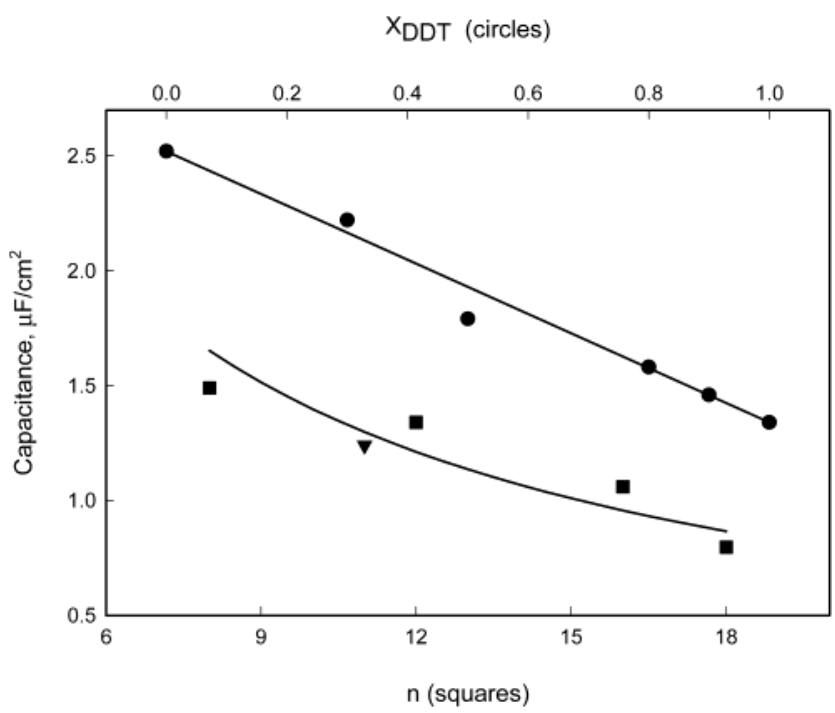

Figure 1. Upper curve - capacitance of the binary HUT/DDT layers as a function of the surface concentration of HUT and DDT thiols. Dots experimental data, line - calculated using equation: $C=C_{0}-X_{\mathrm{DDT}}\left(C_{1}-\right.$ $\left.C_{0}\right)$, where $C_{1}$ is the capacity on $X_{\mathrm{DDT}}=1$, and $C_{0}$ is the capacity on $X_{\mathrm{DDT}}$ $=0$. Lower curve - capacitance as a function of carbon atoms number $n$ of hydrophobic alkylthiols. Squares - experimental data, line calculated using eq 1 with $\epsilon=2.3$ and distance between methylene groups $1.12 \AA$. Triangle corresponds to the fluorinated compound.

capacitance values for the single-component hydrophobic surfaces are in very good agreement with published data. ${ }^{30-32}$ For the shorter alkanethiols, larger capacitance values have been attributed to packing defects. ${ }^{30}$ Small deviations from the literature values for the shorter alkanethiols and the fluorinated compound $^{33}$ can be attributed to differences in surface roughness and methods of surface preparation. The capacitance of the HUT layer $\left(2.5 \mu \mathrm{F} / \mathrm{cm}^{2}\right)$ is somewhat larger than the data published by Chidsey $1.8 \mu \mathrm{F} / \mathrm{cm}^{2}{ }^{33}$ and Miller $2.01 \mu \mathrm{F} / \mathrm{cm}^{2},{ }^{34}$ but some of this difference may be due to the solvent used in preparation. ${ }^{34}$ The capacitance of the binary DDT/HUT surfaces increases linearly (Figure 1) with increasing HUT surface concentration. This suggests the absence of concentration-dependent phase separation and that concurrent addition of the defects in the monolayers are not occurring. To verify and confirm the absence of phase separation on the binary surfaces, we carried out a reductive desorption experiment on pure DDT, pure HUT, and 1:1 DDT/HUT samples (data not shown). Reductive desorption of the pure HUT and DDT films showed that the desorption potentials differ slightly, as expected, with the DDT desorbing at a potential of approximately $30 \mathrm{mV}$ greater than the desorption potential for the HUT. The 1:1 mixed monolayer desorbed at a potential between the two, and the desorption peak showed some broadening in comparison to the pure monolayers. The data provide no evidence for domain formation in these binary monolayers. This is in accordance with experiments ${ }^{25}$ in which binary monolayers were assembled from THF and is distinctly different from the case of binary mixtures formed from ethanol which give two desorption peaks. ${ }^{35}$

(30) Porter, M. D.; Bright, T. B.; Allara, D. L.; Chidsey, C. E. D. J. Am. Chem Soc. 1987, 109, 3559-3566.

(31) Widrig, C. A.; Chung, C.; Porter, M. D. J. Electroanal. Chem. 1991, 310, $335-359$.

(32) Boubour, E.; Lennox, R. B. Langmuir 2000, 16, 4222-4228.

(33) Chidsey, C. E. D.; Loiacono, D. N. Langmuir 1990, 6, 682-691.

(34) Miller, C.; Cuendet, P.; Gratzel, M. J. Phys. Chem. 1991, 95, 877-886.

(35) Bain, C. D.; Evall, J.; Whitesides, G. M. J. Am. Chem. Soc. 1989, 111, $7155-7164$

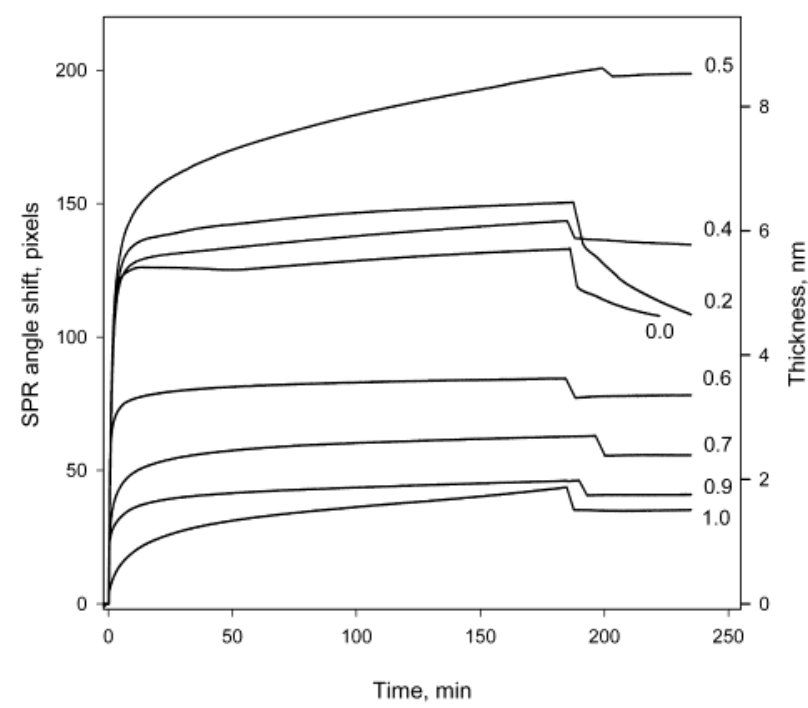

Figure 2. SPR kinetics of POPC adsorption at the binary HUT/DDT surface. Lines are annotated by numbers that indicate the molar fraction $X_{\mathrm{DDT}}$ of dodecanethiol. Breaks in the smooth lines indicate the start of flow of buffer solution without vesicles.

SPR, Capacitance, and AFM Data for POPC Association with Binary Surfaces. Figure 2 shows a time course for POPC adsorption at the binary thiol surfaces using the SPR technique. Before the start of the kinetics measurements, the baseline was recorded in buffer solution for $15 \mathrm{~min}$. At the beginning of the measurement (time $=0 \mathrm{~min}$ ), the entire volume of the SPR cell was substituted with buffer solution containing POPC vesicles. As seen in Figure 2, POPC adsorbed to all binary thiol surfaces. After $3 \mathrm{~h}$ of adsorption time, vesicles were removed from the cell by a continuous flow of buffer. The smallest change in optical thickness was detected for the surface containing only DDT $\left(X_{\mathrm{DDT}}=1\right)$, and the largest change occurred for $X_{\mathrm{DDT}}=0.5$. The difference in SPR response before and after rinsing the cell is due to differences in the refractive index of the buffer with and without vesicles and also due to desorption from the surface of loosely associated POPC molecules. For two of the surfaces, pure HUT and the surface with $X_{\mathrm{DDT}}=0.2$, substantial amounts of the POPC were desorbed from the surfaces during the 60 min rinse with buffer. It is also apparent from Figure 2 that the rate of POPC adsorption at the pure DDT surface was slower than it was at the other surfaces. The SPR data for the pure hydrophilic surface $\left(X_{\mathrm{DDT}}=0\right)$ show a maximum in thickness change after a fast adsorption step, followed by a decrease in thickness during the following $50 \mathrm{~min}$ period of incubation in POPC solution (Figure 2 , curve 0.0 ).

The differences in capacitance before and during POPC vesicle addition to the binary thiol surfaces are shown in Figure 3 . If the dielectric constant, $\epsilon$, of a material is known, the capacitance, $C$, provides a measure of the thickness, $d$, of the dielectric layer:

$$
1 / C=d / \epsilon \epsilon_{0}
$$

As shown in Figure 1, the capacitance of the DDT monolayer was measured to be $1.5 \mu \mathrm{F} / \mathrm{cm}^{2}$. As a result of the addition of POPC vesicles, the total capacitance of the surface decreased to $0.98 \mu \mathrm{F} / \mathrm{cm}^{2}$, similar to the change observed for the addition of unsaturated phosphatidylcholine to decanethiol monolayers. ${ }^{12}$ 


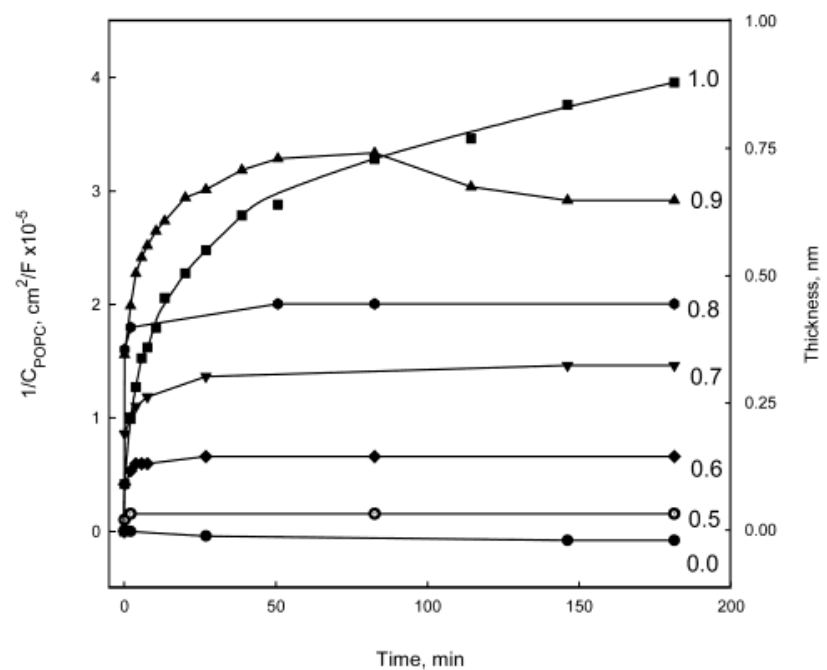

Figure 3. Changes of the inverse capacitance $\left(1 / C_{\mathrm{POPC}}\right.$, see eq 2$)$ during POPC vesicle adsorption onto the binary HUT/DDT surface versus time. Numbers indicate the molar fraction, $X_{\mathrm{DDT}}$, of dodecanethiol.

When a complete insulating layer of POPC adds to an alkanethiol layer to form an HBM, ${ }^{12}$ the thickness of the alkane portion of the POPC layer that adds to the thiol monolayer can be calculated from the capacitance data as follows

$$
1 / C_{\mathrm{POPC}}=1 / C-1 / C_{\text {mono }}
$$

where $C$ is the capacitance of the alkylthiol/POPC bilayer, and $C_{\text {mono }}$ is the capacitance for the monolayer before POPC adsorption. The change in $1 / C$ due to the addition of lipid to the surface is shown in Figure 3. The dielectric constant, $\epsilon$, of a layer of unsaturated phospholipid has been determined to be $2.7 .{ }^{12}$ For the surface composed of $100 \%$ DDT $\left(X_{\mathrm{DDT}}=1\right)$, this analysis indicates that the interaction of POPC vesicles with the surface results in the addition of a dielectric layer with a thickness of $0.8 \mathrm{~nm}$.

As compared to the optical and electrical changes of the pure DDT layer $\left(X_{\mathrm{DDT}}=1\right)$, the binary surfaces composed of both HUT plus DDT showed smaller differences in capacitance but larger changes in optical thickness as measured using SPR. For pure HUT $\left(X_{\mathrm{DDT}}=0\right)$, the surface capacitance did not change at all upon addition of POPC, although SPR results indicate that POPC added to the surface. Negligible changes in capacitance indicate that the lipid that added to the surface did not form an insulating layer.

The structure of the adsorbed POPC layers was also examined using AFM (Figure 4). AFM measurements were carried out in intermittent contact mode on monolayer samples maintained under buffer and treated with POPC using the same protocol as that used for SPR and capacitance measurements. Three surfaces were examined: pure DDT $\left(X_{\mathrm{DDT}}=1\right)$, pure HUT $\left(X_{\mathrm{DDT}}=0\right)$, and $50 \%$ DDT/HUT $\left(X_{\mathrm{DDT}}=0.5\right)$. The surfaces were scanned prior to POPC addition, and again after $1 \mathrm{~h}$ of incubation with POPC solution in the fluid cell. Figure 4 shows images of the surfaces after POPC adsorption. Graphs show a selected line scan for surfaces before adsorption (thin line) and after adsorption (thick line). For the pure DDT surface (Figure 4a), AFM indicates a very smooth surface following POPC adsorption. Comparison of the two line scans shows that the surface is approximately as smooth after addition of POPC as the monolayer is before addition of POPC.
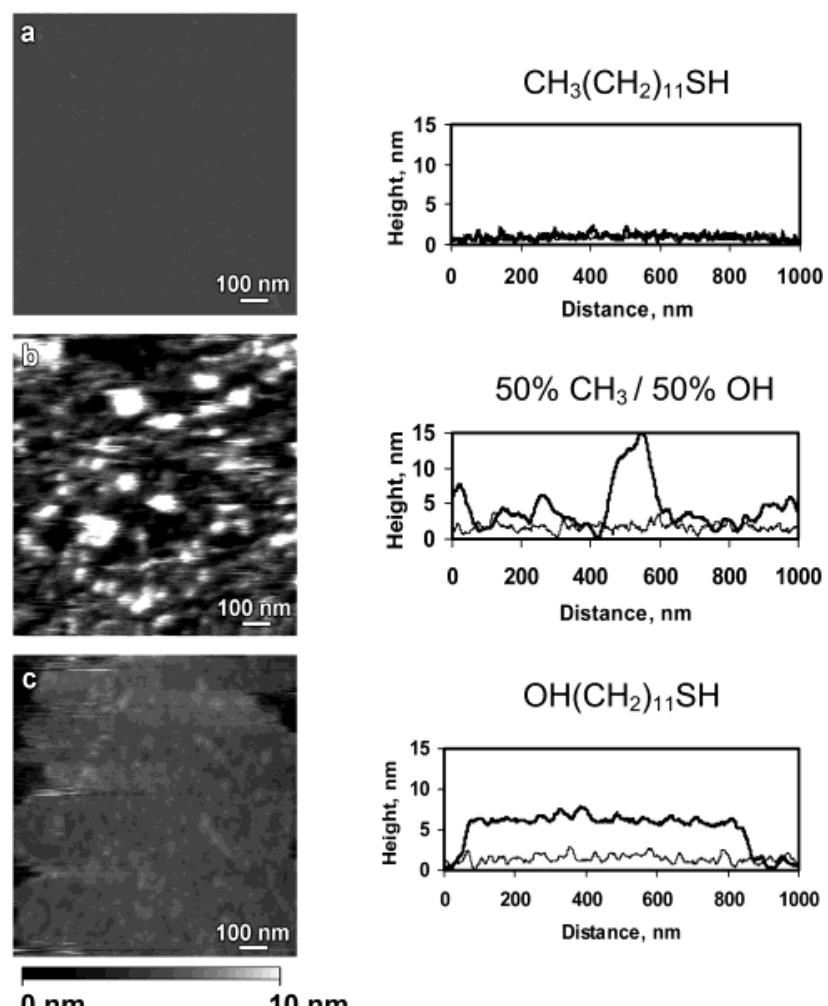

$0 \mathrm{~nm}$

$10 \mathrm{~nm}$

Figure 4. AFM images of the binary surfaces after POPC adsorption. Selected line scans of the surfaces before POPC adsorption (thin line) and after (thick line) are shown. The zero point of the height scale was set to the lowest point in each scan line. Images were collected with vesicles in the fluid cell. (a) 100\% DDT monolayer and associated POPC; (b) binary surface with 50\% HUT and 50\% DDT and associated POPC; (c) 100\% HUT monolayer and associated POPC.

In contrast, the surface containing $50 \%$ of HUT, $X_{\mathrm{DDT}}=0.5$ (Figure 4b), looks very different before and after POPC adsorption. AFM shows a heterogeneous surface with features that are tens of nanometers in diameter, and up to $15 \mathrm{~nm}$ in height following association with vesicles. This result is consistent with the SPR data, which show that a large amount of POPC adsorbs at that surface. At the pure HUT surface, initial AFM scans after POPC addition failed to produce meaningful, consistent images. Scanning with a small $(\sim 1 \mathrm{nN})$ applied force produced a series of images in which a region that was higher and produced more stable images formed and grew to fill the scan area. The image shown (Figure 4c) is the second of four images and shows the stable, higher region in the center of the scan. Scanning a larger area with the original stronger force indicated that the area outside of the area shown in the figure still appeared disordered, while the square from the original scan area imaged well and was $5 \mathrm{~nm}$ higher than the surrounding area. We hypothesize that with a moderate force, the AFM tip was capable of penetrating the lipid layer, and that scanning with a very low force resulted in improving the molecular organization of the layer. A similar phenomenon has been reported by Santos et al., ${ }^{36}$ who observed the healing of a defect in a DPPE bilayer upon scanning with the AFM, leaving large defect-free areas amid the highly defective original bilayer. The total thickness of the lipid added to the SAMs could not be determined by AFM because the level of the SAM could not

(36) Santos, N. C.; Ter-Ovanesyan, E.; Zasadzinski, J. A.; Catanho, M. Biophys. J. 1998, 75, 2119-2120. 


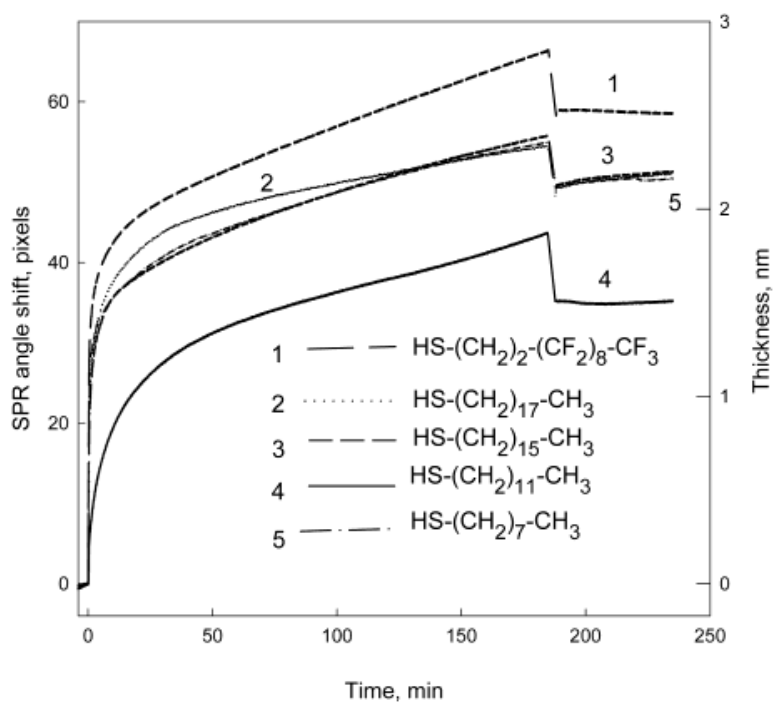

Figure 5. SPR kinetics of POPC adsorption at hydrophobic surfaces of different length alkanethiols and a fluorinated thiol. Designations indicate the carbon chain lengths of the molecules.

be unambiguously determined once the POPC was adsorbed. However, combining the SPR data with AFM data allows us to conclude that the pure DDT and HUT surfaces have a uniform monolayer and bilayer of POPC, respectively, while the 50/50 surface consists of a heterogeneous lipid layer that may include multilayered areas and intact vesicles.

Interaction of POPC with Single-Component Hydrophobic Surfaces. To examine in greater detail the HBM formation at the lowest level of the polar part of the surface energy, we also investigated the interaction of phospholipid vesicles with single-component hydrophobic surfaces composed of methylterminated thiols of different numbers, $n$, of carbon atoms. Lingler et al. ${ }^{17}$ found that with decreasing $n$, the optical thickness of the phospholipid layer that was added increased, especially for $n<9$. Conversely, capacitance data indicated that with decreasing $n$, the lipid layer became less insulating. The observation that a large optical thickness increase is accompanied by a low electrical thickness is analogous to our observations with binary surfaces. To directly examine this similarity, we measured the kinetics of HBM formation on four methyl terminated thiol monolayers of $n=8,12,16$, and 18, and a fluorinated alkanethiol with $8 \mathrm{CF}_{2}$ groups and $2 \mathrm{CH}_{2}$ groups. SPR data are shown in Figure 5, capacitance data are shown in Figure 6, and contact angles and surface free energies for these monolayers are shown in Table 2. The contact angle of water was greatest for the fluorinated compound and decreased with decreasing $n$, in good agreement with literature data. ${ }^{37}$ After $3 \mathrm{~h}$ of incubation with POPC vesicles, the largest amount of POPC adsorbed on to the fluorinated compound, while the smallest amount of POPC adsorbed onto the monolayer with $n=12$. For $n=16,18$, and 8 , the kinetic curves and final thickness of POPC are similar. For all of the surfaces, the adsorption of vesicles results in an optical thickness between 1.5 and $2.5 \mathrm{~nm}$. For the single-component surfaces, the increase in SPR response with time does not saturate during $3 \mathrm{~h}$ in the presence of POPC vesicles. Wenzl and co-workers ${ }^{38}$ did not

(37) Bain, C. D.; Whitesides, G. M. Langmuir 1989, 5, 1370

(38) Wenzl, P.; Fringeli, M.; Goette, J.; Fringeli, U. P. Langmuir 1994, 10, $4253-4257$

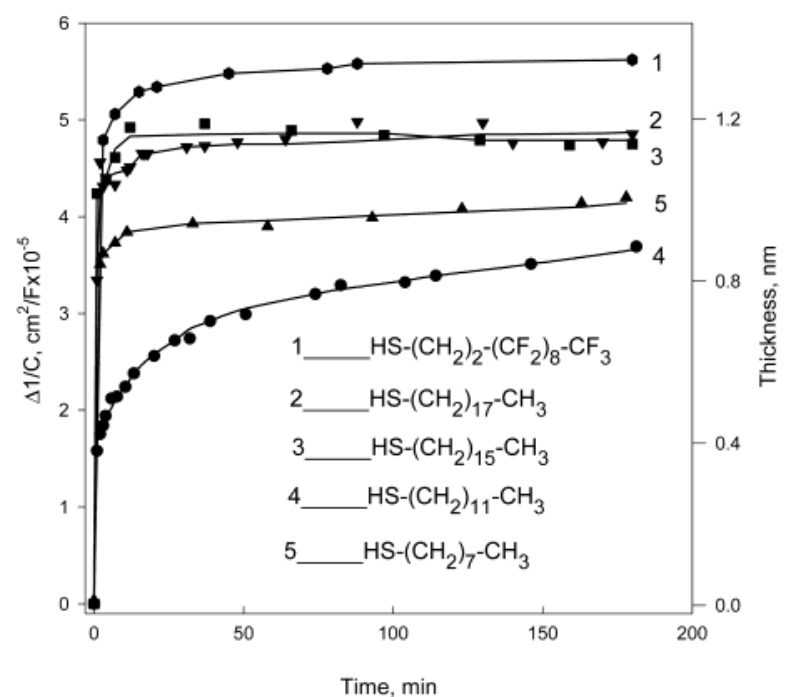

Figure 6. The inverse capacitance $\left(1 / C_{\mathrm{POPC}}\right.$, see eq 2$)$ before and during POPC vesicle adsorption at hydrophobic surfaces versus time. Thickness was calculated using eq 1 assuming $\epsilon=2.7$. Each line represents the data from two to four replicate experiments.

observe saturation for POPC adsorption even after $30 \mathrm{~h}$ of incubation time. The sharp drop in the SPR response after rinsing out the vesicle solution (Figure 5) corresponds to the difference between the refractive index of the buffer solution and the buffer solution containing POPC vesicles, plus the loss of loosely associated vesicles from the surface. Most likely, the addition of the lipid layer has two kinetic regimes. The first regime indicates POPC monolayer addition (within the first 20 or $30 \mathrm{~min}$ ), which is followed by a slow change that can be attributed to the adsorption of POPC molecules or vesicles to the HBM surface.

Capacitance measurements as a function of time during POPC layer addition are presented in Figure 6. Contrary to the SPR data (Figure 5), the apparent kinetics of the addition of the POPC layer as determined by capacitance measurements do reach a limiting value. This difference suggests that the additional POPC that adheres to the HBM does not form an insulating layer. Similar to the SPR data, the apparent thickness of the lipid layer is smallest when the monolayer is dodecanethiol $(n=12)$, and the largest change is associated with the fluorinated compound. The curves for POPC addition to $n=16$ and $n=18$ overlap. However, the magnitude of the change in capacitance due to POPC addition to the $n=8$ monolayer is not consistent with the relative SPR response. Optical thickness measurements indicate that the POPC layer that forms at $n=8$ is equivalent to that formed at the $n=16$ and $n=18$ monolayers; however, the change in capacitance indicated that the POPC layer is thinner on $n=8$. The difference in apparent optical and electrical thickness of the POPC layer formed at the different monolayers suggests that the structure of the lipid layer is different on these different underlying monolayers.

On the basis of a simple chemical model, we estimate that the maximum length of the fully trans extended methylene part of the POPC molecule is $\sim 2.0 \mathrm{~nm}$ and the size of the headgroup is $\sim 0.7 \mathrm{~nm}$. These dimensions would indicate a potential film thickness that is much larger than is determined by our measurements (Figures 5 and 6). The apparent thicknesses we determine suggest a high degree of disorder in the POPC molecules, which is not unexpected due to the presence of the 


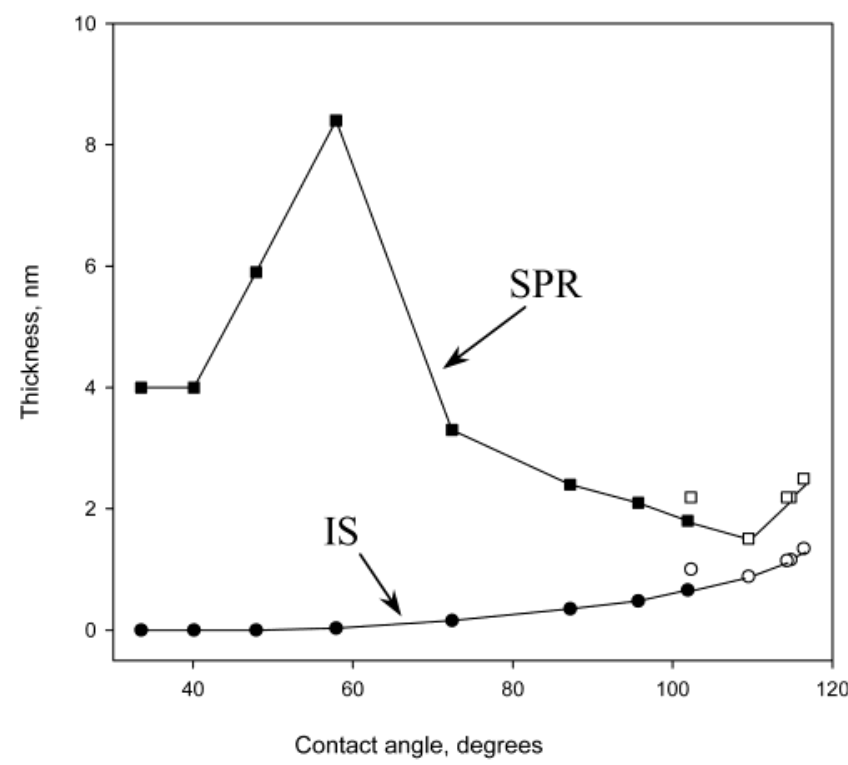

Figure 7. Plot of apparent capacitive thickness as determined from impedance spectroscopy $(\bullet)$ and apparent optical thickness as determined from SPR (ם) versus contact angle. $\square$ and $\bigcirc$ correspond to the single hydrophobic surfaces, and and refer to the binary DDT/HUT surfaces. The curves through the data are to guide the eye. (Refer to Tables 1 and 2 for surface free energy, $X_{\mathrm{DDT}}$ ratio, or alkylthiol chain lengths for every presented point.)

double bond in the oleyol lipid chain and the fact that the POPC is in the fluid phase at temperatures above $-5{ }^{\circ} \mathrm{C}$. In addition, capacitance measurements are sensitive only to the methylene (hydrophobic) part of the phospholipid molecules because the headgroup moiety is highly hydrated. ${ }^{39}$ Another cause of the apparent differences in thickness that result from optical measurements (Figure 5) as compared to the corresponding electrical measurements (Figure 6) is the tendency for the addition of an overlayer of POPC to form on top of the HBM. The SPR technique detects the total amount of material that adsorbs on the surface, ${ }^{40}$ while the impedance technique is sensitive only to electrically insulating layers.

\section{Discussion}

Figure 7 plots the data obtained by SPR and capacitance after $3 \mathrm{~h}$ following addition of phospholipid vesicles into SPR cell. These data are plotted versus the water contact angle of the monolayer surfaces. As one can see, the SPR data show a maximum around a contact angle of 60 and a minimum at around a 108 contact angle. The SPR curve (Figure 7) can be divided in three distinct regions: below a contact angle of 40, which corresponds to hydrophilic surfaces that are characterized by a high value of the polar part of the surface energy (Figure 8 , Table 1); above a contact angle 108, which corresponds to hydrophobic surface with a low value for the polar part of the surface energy (Table 2); and the region of contact angles between 40 and 108 where the maximum in SPR response occurs (Figure 8, Table 1). Below we will briefly discuss the phospholipid layers addition in these regions.

POPC Interaction with Hydrophilic Surfaces. At the surface composed of $100 \% \mathrm{OH}$ terminated thiol $\left(X_{\mathrm{DDT}}=0\right)$, we observe a change in thickness that is equivalent to the addition of a bilayer of phospholipid. This is not unexpected

(39) Benz, R.; Frohlich, O.; Lauyer, P.; Montal, M. Biochim. Biophys. Acta 1975, 394, 323-334.

(40) Silin, V.; Plant, A. L. Trends Biotechnol. 1997, 15, 353-359.

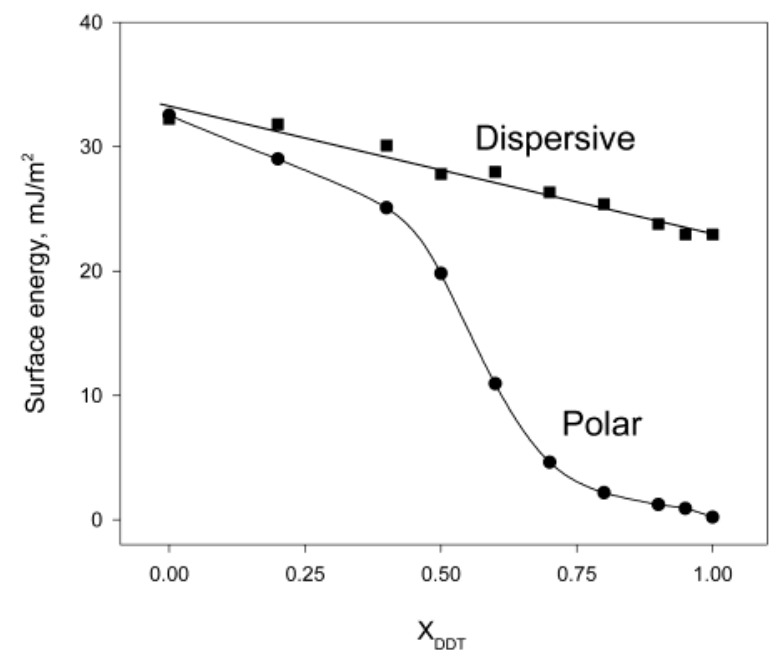

Figure 8. Dependence of the dispersive and polar parts of surface energy on the surface concentration of DDT thiol.

because the underlying monolayer surface is hydrophilic. For hydrophilic surfaces, vesicle adsorption, rupture, and addition of a lipid bilayer are well known and have been extensively studied by Seifer and Lipowsky ${ }^{19,41-44}$ and recently by Kasemo and Zhdanov. ${ }^{45-47}$ In general, the driving force for vesicle rupture and fusion at a hydrophilic surface is the surface adhesion energy. ${ }^{18,19}$ The strong interaction between the surface and an adsorbed vesicle leads to vesicle deformation stress, pore formation, and, finally, to fusion between vesicles and addition of a bilayer to the hydrophilic surface. For a given adhesion energy, there is a critical local radius of curvature in the vesicle at which rupture will occur. ${ }^{45}$

POPC Interaction with Hydrophobic Surfaces. For hydrophobic surfaces, there is still little known about the initial steps in the interaction, even though a number of studies have examined kinetics and other aspects of vesicle reorganization at these surfaces. ${ }^{1}$ Although the hydrophobic surface in contact with water likely provides the thermodynamic driving force for addition of a monolayer of phospholipid, the rate-limiting step in the process of disruption and reorganization of the lipid vesicle is unknown. It seems unlikely that hydrophobic alkyl moieties of the phospholipid would be exposed to water in the process of adding a lipid monolayer to the surface because such exposure would represent a large thermodynamic barrier. Nevertheless, the process occurs very rapidly under ambient conditions. Using the quartz crystal microbalance technique, Keller et al. ${ }^{48}$ showed that a major difference between phospholipid monolayer addition on a hydrophobic surface and phospholipid bilayer addition on a hydrophilic surface is that in the former case, no adsorption of vesicles to the surface can be discerned.

In this current study with POPC vesicles, SPR and impedance data indicate (Figure 7) the addition of a monolayer of phospholipid (i.e., the formation of $\mathrm{HBM}$ ) at the single-

(41) Seifert, U. Phys. Rev. A 1991, 43, 6803-6814.

(42) Seifert, U.; Lipowsky, R. Phys. Rev. A 1990, 42, 4768-4771.

(43) Lipowsky, R.; Seifert, U. Langmuir 1991, 7, 1867-1873.

(44) Lipowsky, R. Festkorperprobleme - Advances in Solid State Physics 32; 1992; Vol. 32, pp 19-44.

(45) Zhdanov, V. P.; Kasemo, B. Langmuir 2001, 17, 3518-3521.

(46) Zhdanov, V. P.; Keller, C. A.; Glasmastar, K.; Kasemo, B. J. Chem. Phys. 2000, 112, 900-909.

(47) Keller, C. A.; Glasmastar, K.; Zhdanov, V. P.; Kasemo, B. Phys. Rev. Lett. 2000, 84, 54443-55446.

(48) Keller, C. A.; Glasmastar, K.; Zhdanov, V. P.; Kasemo, B. Phys. Rev. Lett. 2000, $84,5443-5446$. 
component thiols of $\mathrm{CF}, \mathrm{C} 18, \mathrm{C} 16$, and $\mathrm{C} 12$, accompanied by a small amount of additional POPC overlayer. It is necessary to note the differences in the apparent optical and electrical thickness of POPC on the single-component alkane surfaces (Figure 7, $\square$ and $\bigcirc$ ), which suggest that the packing density of the POPC component of these HBMs is different on these different underlying monolayers. It has been observed that as the chain length or the number of methylene groups of the alkylthiols decreases (especially for $n<9$ ), the structure of the alkylthiol monolayer becomes increasingly disordered, which is indicative of low packing density, ${ }^{17,30}$ domain formation, and thinner regions in the domain boundaries. ${ }^{30}$ These characteristics would result in a shorter path between ions in solution and the underlying electrode surface, making these films less insulating than would be estimated based on the theoretical thickness of the monolayer and most likely will lead to an increase in the polar part of the surface energy (Table 2). The fluorinated compound used in this study has the largest contact angle, the best insulating properties, and the smallest polar part of surface energy (Table 2). While the molecule is not as large as the $n=$ 18 alkanethiol, it is oriented almost normal to the surface, ${ }^{30,33}$ while the protonated compounds have a chain tilt of $\sim 30^{\circ}$ from normal. A small tilt angle of the fluorinated compound leads to better packing of molecules at the surface and results in a smaller number of defects in the thiol layer. ${ }^{33}$

If we examine the density of the added POPC layer, we find that POPC density is higher on monolayer surfaces with a smaller number of defects. On the basis of the values measured for electrical thickness, we can estimate the areas occupied by each POPC molecule in these HBMs. If we assume that the volume of a POPC molecule is $1263 \AA^{3}, 49,50$ and that the headgroup occupies approximately $30 \%$ of the total POPC volume (based on molecular weight), we can calculate from the electrical thickness measurements (Figures 6 and 7) the surface area occupied per POPC molecule. This area will be largest on the monolayer of $n=12$ (98 $\AA^{2} /$ POPC), smallest for the fluorinated surface (63 $\AA^{2} / \mathrm{POPC}$ ), and equal (74 $\AA^{2} / \mathrm{POPC}$ ) for $n=16$ and $n$ $=18$ surfaces. The area calculated for POPC on the fluorinated surface is very close to the surface area that POPC occupies in a fully hydrated bilayer, which is $66.4 \AA^{2} /$ POPC. ${ }^{50}$ These values for molecular area indicate that POPC is more densely packed on the fluorinated surface than on the $n=12$ surface, suggesting that phospholipid order in HBMs correlates with order and packing density of the underlaying alkanethiol monolayer. These monolayer characteristics may also influence the kinetics of HBM formation. As shown in Figure 6, HBM formation on the $n=12$ surface is much slower than for other surfaces, does not reach saturation in $3 \mathrm{~h}$, and results in a thinner HBM.

Intermediate Region of the Polar Part of the Free Energy. While phospholipid vesicles appear to add a lipid monolayer to single-component alkane surfaces of $n \geq 12$, and a bilayer to the fully $\mathrm{OH}$ terminated surface, it is less clear what occurs at the surfaces with contact angles less than 109.5. Comparison of SPR and capacitance data (Figure 7) suggests that at the C8 surfaces and the binary HUT/DDT surfaces, the association of POPC vesicles with the surfaces results in more POPC adding to the surface, but as a noninsulating structure. The amount of POPC molecules on the surface significantly increases for $X_{\mathrm{DDT}}$

(49) Nagle, J. F.; Weiner, M. C. Biochim. Biophys. Acta 1988, 942, 1-10. (50) Chui, S. W.; Jackobsson, E.; Subramaniam, S.; Scott, H. L. Biophys. J. 1999, $77,2462-2469$. concentration around $50 \%$ and decreases below and above this concentration (Figure 7). Interestingly, in the same $X_{\mathrm{DDT}}$ concentration region, the surface energy changes dramatically (Figure 8 ). To understand the behavior of the polar part of the surface energy (Figure 8) of the binary surfaces, Ulman proposes the wetting transition model. ${ }^{26}$ On the basis of this model, on binary surfaces POPC vesicles interact with surfaces that are mixtures of both hydrogen bonding and hydrophobic areas. Presumably, at low $\mathrm{OH}$-containing surfaces, vesicles interact with the surface predominantly via hydrophobic interactions, adding mostly a monolayer of lipid to the surface, but with some stabilization of lipid vesicular structure by occasional patches of hydrogen bonding. At intermediate concentrations of $\mathrm{OH}$ groups $\left(X_{\mathrm{DDT}}\right.$ $\approx 0.5$ ), that correspond to the wetting transition region ${ }^{26}$ (Figure 8 ), the apparent thickness of the POPC layer is maximum. It is within this transition region that the arresting of vesicle fusion takes place most noticeably. We hypothesize that the prewetting layer described by Ulman stabilizes vesicles, preventing their reorganization and addition of a monolayer of lipid, but does not provide sufficient adhesion energy to drive formation of a complete POPC bilayer. As a result, partially fused vesicles are trapped at the surface. AFM data (Figure 4) suggest that the structures at the surface may include some intact vesicles. It is likely that the AFM tip deforms vesicles adsorbed to the surface, so that the actual size of the features may be greater than the measured $15 \mathrm{~nm}$. However, it also appears from the AFM image that vesicles are partially fused with the surface.

\section{Conclusions}

In this study, we have examined the influence of the surface free energy of underlying monolayers on phospholipid vesicles interaction with those surfaces. The surface free energy of the underlying monolayer was changed in a systematic predictable manner in the widest possible range of contact angles using mixed DDT/HUT alkanethiols or using alkanethiols of different chain lengths. Other parameters such as surface roughness, POPC vesicle size, temperature, and vesicle concentration were kept constant. Comparison of capacitance and SPR data allows us to draw the conclusion that for single-component hydrophobic surfaces, the POPC layer structure may depend on the alkylthiol chain length and tilting angle, which determines the packing density of the monolayer. For binary surfaces of intermediate free energy, the existence of both hydrophobic groups and hydrogen bonding groups complicates the interaction of the vesicles with the surface. The binary surface partially stabilizes vesicles, arresting their reorganization and addition of a monolayer of lipid, while it does not provide sufficient adhesion energy to drive rupture and addition of a bilayer of lipid. Importantly, poorly ordered single-component alkanethiol surfaces (e.g., $n=8$ ) can be classified with the lower surface energy binary systems. The data presented here suggest that success in HBM formation at a hydrophobic surface will be dependent on the extent of the polar component of the surface free energy of that surface. Even for surfaces with a high (ca. $100^{\circ}$ ) contact angle with water, the presence of even small amounts of $\mathrm{H}$-bonding can stabilize the vesicle structure and prevent the addition of a homogeneous monolayer of lipid to the surface.

Acknowledgment. We wish to thank Dr. Lee Richter for useful discussions.

JA026585+ 